\title{
Contralateral non-simultaneous proximal femoral fractures in patients over 65 years old
}

\author{
Francesco Bosco $^{1}$ (D) Jacopo Vittori ${ }^{1} \cdot$ Elena Grosso $^{1} \cdot$ Mariapaola Tarello $^{1} \cdot$ Stefano Artiaco $^{1} \cdot$ Alessandro Massè $^{1}$
}

Received: 11 January 2021 / Accepted: 6 March 2021 / Published online: 17 March 2021

(c) The Author(s) 2021

\begin{abstract}
Purpose Epidemiological and clinical parameters according to the Parker-Palmer Index (PPI) have not been specifically studied as predictors of re-fracture time in patients over 65 years old with contralateral hip fracture. The main purpose of this study was to assess whether these parameters could represent a prognostic factor in this population.

Methods This retrospective study included all consecutive patients older than 65 years that suffered from a proximal femoral fracture, 31 according to Association for Osteosynthesis/Orthopaedic Trauma Association classification, treated at our unit between Feb 1st 2019 and Feb 1st 2020.

Results This study enrolled 387 patients. Thirty-seven of them had already incurred a contralateral hip fracture: seven males and 30 females. The median time between the first and second hip fractures was 3.5 years. This study revealed that increasing age $(p=0.003)$, male sex $(p=0.029)$ and a PPI value $\geq 5$ between the first and second hip fracture $(p=0.015)$ are risk factors associated with a contralateral hip fracture in the first three years after the first episode. There were no statistically significant differences regarding anti-osteoporotic therapy and the anatomic site of the first hip fracture episode.

Conclusion The results of the present study suggest that several risk factors have a crucial role in hip re-fracture time in patients over 65 years old.
\end{abstract}

Keywords Contralateral hip fracture $\cdot$ Proximal femoral fracture $\cdot$ Risk factor $\cdot$ Re-fracture time

\section{Introduction}

Proximal femoral fractures are among the main causes of disability in the elderly. A large proportion of the patients who undergo a femoral fracture lose part of their independence in daily activities, becoming bedridden, suffering from prolonged hospitalization, needing long-term care in facilities or requiring a walking aid. Another important factor to consider is the mortality rate in this population: $10 \%$ at one month from surgery and up to $35 \%$ at the end of the first year [1-3].

A hip fracture is often the initial presentation of underlying osteoporosis and affected patients should be considered at risk of developing other fragility-related fractures, including those of the contralateral hip. According to a recent study by Balasubramanian et al., most of the contralateral

Francesco Bosco

francescobosco@icloud.com

1 Department of Orthopaedics and Traumatology, University of Torino, Via Zuretti, 29, 10126 Turin, Italy fractures occur within a 12-month timespan [4]. There is a marked discrepancy between authors regarding the occurrence rate of contralateral hip fracture, with some reporting an incidence of $2.7 \%$ at one year and approximately $7.8 \%$ at 8.5 years [5]. Other studies concluded that the cumulative incidence is approximately $9 \%$ at one year and $20 \%$ after five years [6]. The occurrence of contralateral fractures may be related to the mortality rate in these patients. According to different authors, mortality rates at one and five years were 15.9 and $45.4 \%$, respectively, whereas mortality rates in patients who suffered previously from contralateral hip fracture were 24.1 and $66.5 \%$, respectively, at one and five years since the second event $[7,8]$. The fracture site and pattern of the second episode on the contralateral limb appeared to be similar to that of the first episode [9].

Risk factors for contralateral hip fractures have been thoroughly assessed. Juhàsz et al. [10] demonstrated that living in a capital city, hip arthroplasty after the first episode, female sex and older age were all risk factors for a contralateral hip fracture. A meta-analysis by Zhu et al. [11] indicated a higher risk of contralateral hip fracture in patients who 
were female, living in institutions, visually impaired, living with dementia or Parkinson's disease or suffering from cardiovascular or respiratory diseases. Identification of highrisk groups is crucial for the development of preventive strategies. Fast mobilization after hip fracture is one of the most important factors affecting mortality after surgery.

In some studies, the PPI has proven to be a valid measurement of hip fracture patient mobility and a predictor of mortality with high inter-test reliability $[12,13]$. The index is a measurement of the patient's mobility indoors, outdoors and during shopping that can be easy to investigate at hospital admission and follow-up controls [14, 15]. Furthermore, Pedersen et al. demonstrated a strong correlation of the Parker-Palmer Index with other functional scores (Barthel-20 and Barthel-100) concerning gait function prediction [16]. Mobility score has not been specifically studied in contralateral hip fracture patients as a predictor of re-fracture time. The aim of this study was to retrospectively evaluate epidemiological and clinical parameters according to ParkerPalmer Index. Furthermore, we defined the temporal role they played in a contralateral episode of hip fracture. This finding that has never been investigated in the literature was examined in a consecutive series of patients treated in our unit for one year.

\section{Methods}

We conducted a retrospective study including 387 consecutive patients over 65 years old. All patients suffered from a proximal femoral fracture, 31 according to Association for Osteosynthesis/Orthopaedic Trauma Association (AO/ OTA) classification, treated at our Orthopaedics and Trauma Department between Feb 1st 2019 and Feb 1st 2020. Every radiograph has been evaluated and the fractures have been classified by two independent reviewers to reach concordance about the type of fracture. Any patients for whom the reviewers could not agree on the type of fracture were discarded. We excluded from the study patients with periprosthetic hip fractures, revision hip arthroplasties, simultaneous bilateral hip fractures, polytrauma, oncologic hip fractures, 32, 33 AO/OTA hip fractures in the same period.

Patients have been separated into two groups: Group A ( $n=350)$ consisted of patients who suffered from a single hip fracture, group B $(n=37)$ consisted of patients who suffered from a subsequent contralateral hip fracture. Group B was divided into two subgroups: B1 $(n=18)$ and B2 $(n=19)$, where members of group B1 suffered a contralateral hip fracture within three years of the first episode and members of group B2 suffered a contralateral hip fracture after this timepoint. For each patient, we recorded gender, age at the first hip fracture, type of primary fracture and type of surgical procedure for the first hip fracture. We compared the total deaths in our patient pool between February 1st 2019 and May 1st 2020. Also, specific data were collected for group B patients, such as age at the contralateral hip fracture, initial (at first hip fracture) and final (at contralateral hip fracture) PPI, pattern of contralateral hip fracture, type of surgical procedure for the contralateral fracture, time between first and contralateral hip fracture, ongoing pharmacological therapy for osteoporosis, surgical timing at the first event, return to the original residence and comorbidities: Parkinson disease, dementia, Alzheimer Disease, cardiovascular and cerebrovascular disease, low vision, syncope, epilepsy, dizziness, orthostatic hypotension, respiratory disease. A logistic regression was performed for group B to evaluate the relationship between re-fracture time and epidemiological and clinical parameters. All information was collected by the authors using a standard proforma with direct patient examination, clinical chart evaluation and telephone interview.

Statistical analysis was performed using Statistical Product and Service Solutions (SPSS Inc., Chicago, Illinois). Descriptive statistical analysis was performed for all demographic and clinical data across the entire cohort. Mean and standard deviation and maximum and minimum values were calculated for continuous variables. Absolute number and frequency distribution were calculated for categorical variables. Subsequently, an unpaired Student's $t$ test was used to analyze normally distributed, continuous data. The chisquare test was used to analyse categorical data. Data were collected using a Microsoft Excel spreadsheet (version 2016; Microsoft, Redmond, WA). The odds ratio (OR) was considered statistically significant with a $p$ value $<0.05$.

\section{Results}

A total of 387 proximal femoral fractures were registered at our hospital between Feb 1st 2019 and Feb 1st 2020. In patients with a first hip fracture, there was a female predominance $(n=280,71.14 \%)$. The number of patients who had died by May 1st 2020 was 31 (8.85\%) for group A and three (8.11\%) for group B (Table 1$)$.

Thirty-seven patients among the 387 had already incurred a contralateral hip fracture: seven males and 30 females. The median time between the first and second hip fracture was 3.5 years, ranging from 12 days to 24.67 years (Table 2). Correspondence of anatomic site of contralateral hip fracture was the same as the first hip fracture in 19 cases. All patients from group B were examined for PPI. Before the first hip fracture, the mean value was $8 \pm 1$, between the first and second hip fracture it was $5 \pm 2$, and after the second hip fracture it was $3 \pm 2$. The proportion of patients who received anti-osteoporosis medication before the first hip fracture was lower $(8.11 \%)$ than the proportion who received this medication between their first and second hip fracture 


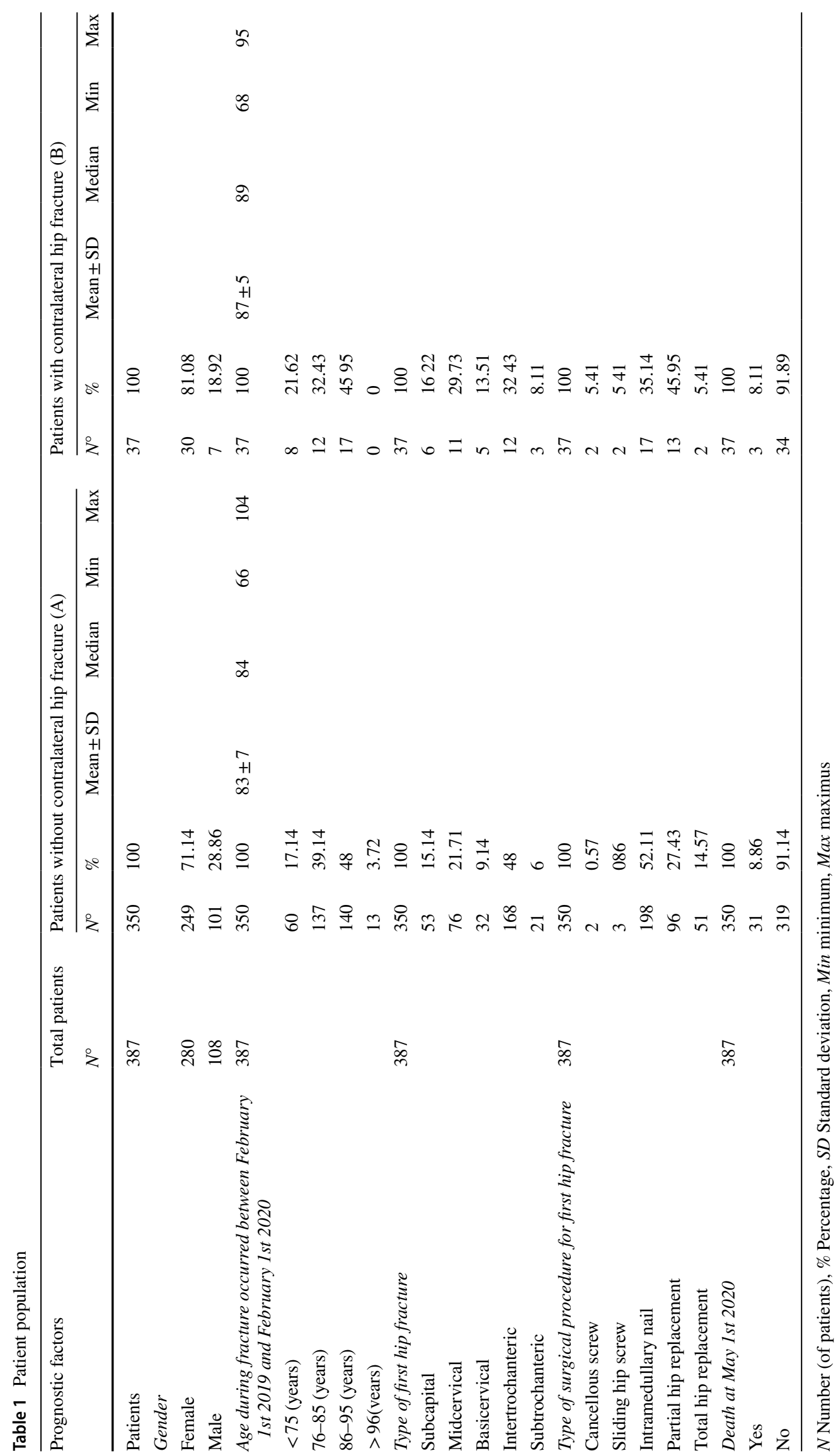


Table 2 Contralateral hip fracture: epidemiological data

\begin{tabular}{|c|c|c|c|c|c|c|c|c|}
\hline \multirow[t]{2}{*}{ Case } & \multirow[t]{2}{*}{ Sex } & \multicolumn{3}{|c|}{ First hip fracture } & \multicolumn{4}{|c|}{ Contralateral hip fracture } \\
\hline & & Age & Type of fracture & Surgical procedure & Age & Type of fracture & Surgical procedure & $\begin{array}{l}\text { Time to second } \\
\text { fracture (days) }\end{array}$ \\
\hline 1 & $\mathrm{~F}$ & 62 & Subtrochanteric & Intramedullary nail & 87 & Midcervical & Partial hip replacement & 9012 \\
\hline 2 & $\mathrm{~F}$ & 57 & Midcervical & Cancellous screw & 80 & Midcervical & Partial hip replacement & 8610 \\
\hline 3 & $\mathrm{~F}$ & 74 & Midcervical & Partial hip replacement & 94 & Midcervical & Partial hip replacement & 7005 \\
\hline 4 & $\mathrm{~F}$ & 62 & Midcervical & Cancellous screw & 78 & Midcervical & Total hip replacement & 5657 \\
\hline 5 & $\mathrm{~F}$ & 73 & Subcapital & Partial hip replacement & 86 & Midcervical & Total hip replacement & 4776 \\
\hline 6 & $\mathrm{~F}$ & 80 & Pertrochanteric & Intramedullary nail & 93 & Pertrochanteric & Intramedullary nail & 4471 \\
\hline 7 & $\mathrm{~F}$ & 78 & Pertrochanteric & Intramedullary nail & 88 & Pertrochanteric & Intramedullary nail & 3880 \\
\hline 8 & $\mathrm{~F}$ & 72 & Midcervical & Partial hip replacement & 84 & Midcervical & Partial hip replacement & 4357 \\
\hline 9 & $\mathrm{~F}$ & 79 & Midcervical & Partial hip replacement & 88 & Pertrochanteric & Intramedullary nail & 3484 \\
\hline 10 & $\mathrm{~F}$ & 72 & Midcervical & Total hip replacement & 82 & Pertrochanteric & Intramedullary nail & 3447 \\
\hline 11 & $\mathrm{~F}$ & 82 & Basicervical & Sliding hip screw & 91 & Pertrochanteric & Intramedullary nail & 3287 \\
\hline 12 & $\mathrm{~F}$ & 82 & Pertrochanteric & Intramedullary nail & 90 & Pertrochanteric & Intramedullary nail & 2865 \\
\hline 13 & $\mathrm{~F}$ & 85 & Subcapital & Partial hip replacement & 91 & Subcapital & Partial hip replacement & 2128 \\
\hline 14 & $\mathrm{~F}$ & 88 & Midcervical & Partial hip replacement & 94 & Pertrochanteric & Intramedullary nail & 1972 \\
\hline 15 & $\mathrm{~F}$ & 84 & Midcervical & Total hip replacement & 89 & Pertrochanteric & Intramedullary nail & 1740 \\
\hline 16 & M & 80 & Basicervical & Sliding hip screw & 84 & Pertrochanteric & Intramedullary nail & 1490 \\
\hline 17 & $\mathrm{~F}$ & 87 & Pertrochanteric & Intramedullary nail & 91 & Subcapital & Partial hip replacement & 1428 \\
\hline 18 & $\mathrm{~F}$ & 86 & Midcervical & Partial hip replacement & 89 & Pertrochanteric & Intramedullary nail & 1279 \\
\hline 19 & $\mathrm{~F}$ & 86 & Subcapital & Partial hip replacement & 89 & Subcapital & Partial hip replacement & 1300 \\
\hline 20 & $\mathrm{~F}$ & 86 & Pertrochanteric & Intramedullary nail & 89 & Pertrochanteric & Intramedullary nail & 810 \\
\hline 21 & $\mathrm{~F}$ & 82 & Midcervical & Total hip replacement & 84 & Subtrochanteric & Intramedullary nail & 848 \\
\hline 22 & M & 89 & Pertrochanteric & Intramedullary nail & 90 & Basicervical & Partial hip replacement & 565 \\
\hline 23 & $\mathrm{~F}$ & 88 & Subcapital & Partial hip replacement & 89 & Subcapital & Partial hip replacement & 559 \\
\hline 24 & $\mathrm{~F}$ & 84 & Midcervical & Partial hip replacement & 86 & Pertrochanteric & Intramedullary nail & 447 \\
\hline 25 & $\mathrm{~F}$ & 91 & Pertrochanteric & Intramedullary nail & 93 & Subtrochanteric & Intramedullary nail & 536 \\
\hline 26 & $\mathrm{~F}$ & 87 & Basicervical & Intramedullary nail & 89 & Pertrochanteric & Intramedullary nail & 747 \\
\hline 27 & $\mathrm{~F}$ & 90 & Basicervical & Partial hip replacement & 92 & Basicervical & Partial hip replacement & 681 \\
\hline 28 & M & 66 & Pertrochanteric & Intramedullary nail & 68 & Midcervical & Total hip replacement & 589 \\
\hline 29 & $\mathrm{~F}$ & 77 & Subcapital & Partial hip replacement & 78 & Pertrochanteric & Intramedullary nail & 474 \\
\hline 30 & M & 87 & Pertrochanteric & Intramedullary nail & 89 & Pertrochanteric & Intramedullary nail & 559 \\
\hline 31 & M & 92 & Pertrochanteric & Intramedullary nail & 93 & Pertrochanteric & Intramedullary nail & 313 \\
\hline 32 & $\mathrm{~F}$ & 86 & Subtrochanteric & Intramedullary nail & 86 & Subtrochanteric & Intramedullary nail & 111 \\
\hline 33 & $\mathrm{~F}$ & 80 & Pertrochanteric & Intramedullary nail & 80 & Pertrochanteric & Intramedullary nail & 144 \\
\hline 34 & $\mathrm{~F}$ & 89 & Basicervical & Intramedullary nail & 89 & Basicervical & Intramedullary nail & 224 \\
\hline 35 & $\mathrm{~F}$ & 95 & Pertrochanteric & Intramedullary nail & 95 & Pertrochanteric & Intramedullary nail & 12 \\
\hline 36 & M & 88 & Subtrochanteric & Intramedullary nail & 88 & Basicervical & Intramedullary nail & 105 \\
\hline 37 & M & 88 & Subcapital & Partial hip replacement & 88 & Subcapital & Partial hip replacement & 59 \\
\hline
\end{tabular}

$M$ Male, $F$ Female

(45.95\%). Moreover, the majority of hip fracture patients were only treated using supplementation with calcium and vitamin D. The prescription of bisphosphonates markedly increased between the two fractures but remained low, with one patient under treatment before the first hip fracture and six patients under treatment after the contralateral hip fracture. One patient treated with denosumab and another treated with teriparatide did not change medical therapy between the first and second hip fracture.

Patients with contralateral hip fracture were divided into two subgroups according to re-fracture time: group B1 consisted of patients who suffered contralateral hip fracture within three years of the first episode, whereas group B2 consisted of patients who suffered contralateral hip fracture over three years after the first episode (Table 3). A male 


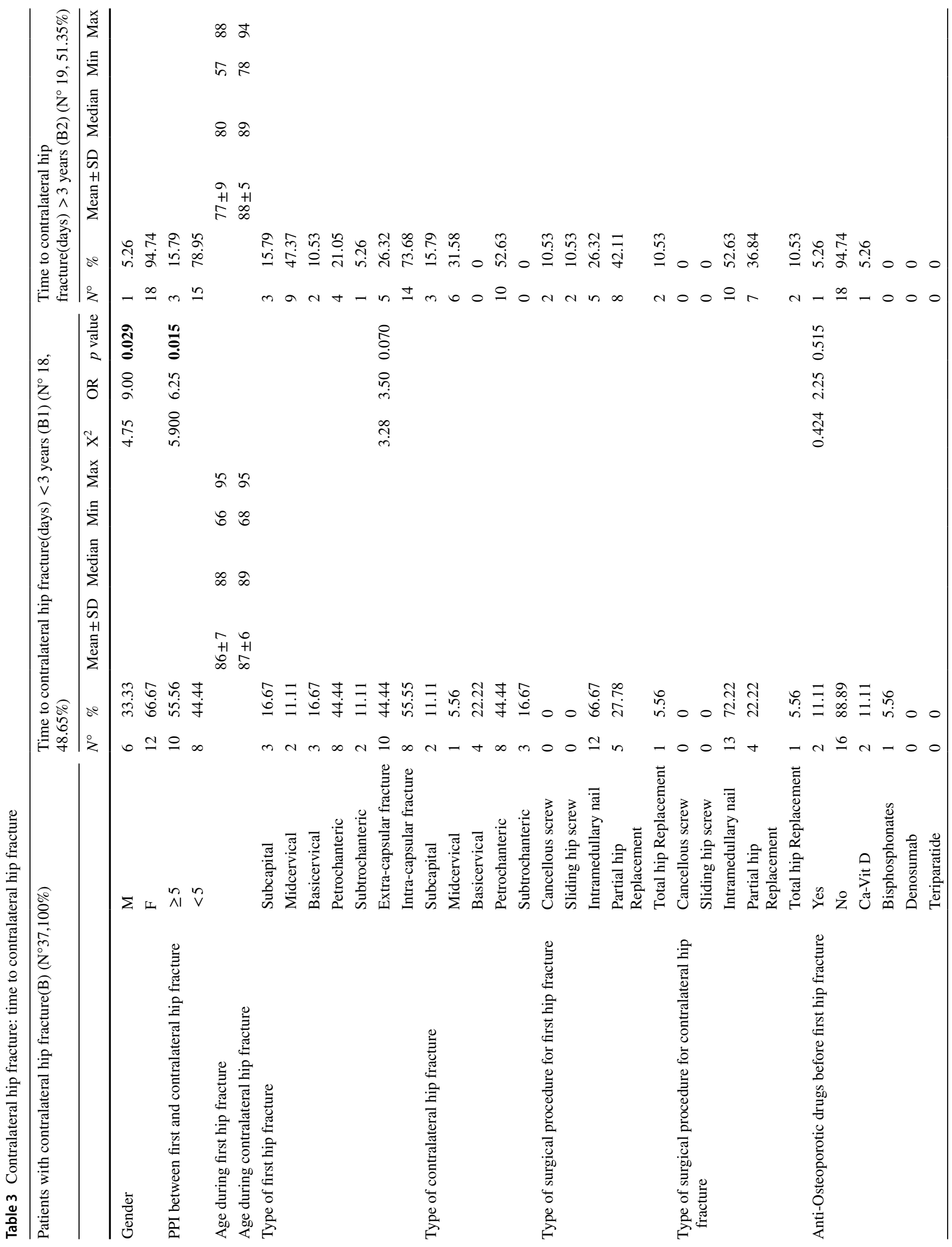




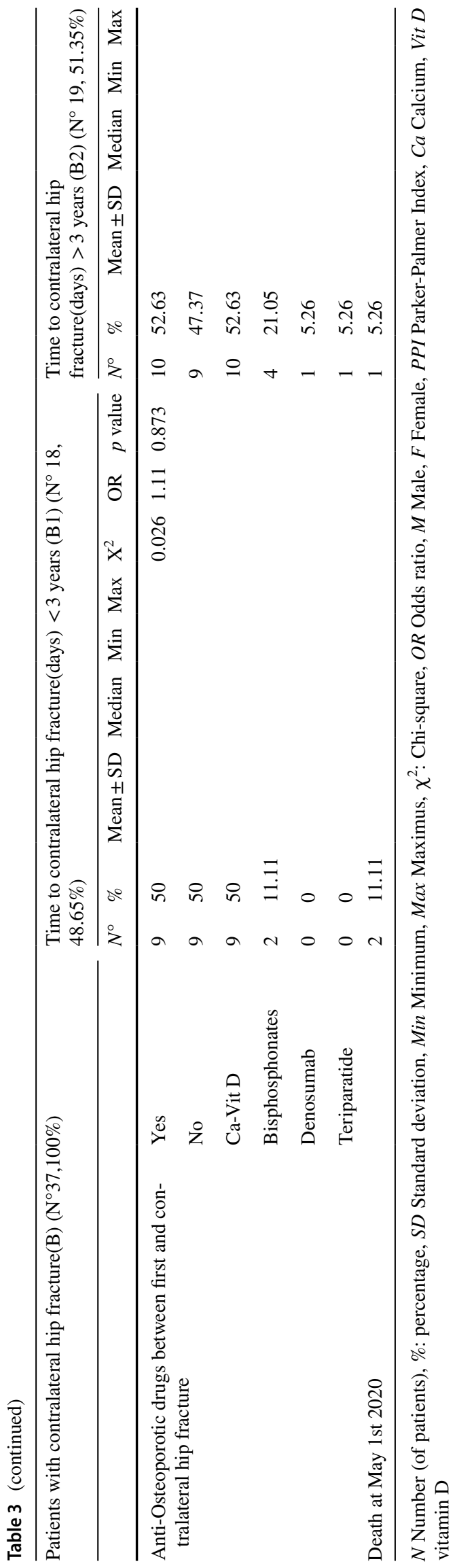

predominance $(n=6,33.33 \%)$ was found in group B1 when compared with group B2 $(n=1,5.26 \%)$. There was a statistically significant difference between the two groups (chi-square: 4.75, OR: 9.00, $p$ value: 0.029 ). The mean age at first hip fracture was $86 \pm 7$ years for the B1 group and $77 \pm 9$ years for the B2 group, whereas contralateral hip fracture patients had a mean age of $87 \pm 6$ years and $88 \pm 5$ years, respectively. These data were analyzed using a t-test and the comparison showed that there was a statistically significant difference between the averages of the two groups. Patients with intra-capsular fractures, compared to those with extracapsular fractures, had a delayed re-fracture time, but this is not statistically significant (chi-square: 3.28 , OR: $3.50, p$ value: 0.070 ). Analysis of PPI of the two groups showed that a value $\geq 5$ was associated with a greater risk of contralateral hip fracture within three years of the first episode. This result was statistically significant (chi-square: 5.90 , OR: $6.25, p$ value: 0.015 ). There was no significant difference between the percentage of patients treated with anti-osteoporotic therapy between the two groups. These data are shown in Table 3.

Therefore, no statistically significant difference $(p$ value $>0.05$ ) in re-fracture timing was found between group B1 and B2 regarding the surgical timing at the first event, return to the original residence and comorbidities. The number of patients who had died by May 1st 2020 was two (11.11\%) for the B1 group and one (5.26\%) for the B2 group respectively. Nevertheless, mortality rate results related to the clinical and epidemiological parameters were not statistically interpretable because our study had a short time of follow-up.

\section{Discussion}

Analysis of our data showed that the incidence of contralateral femoral fractures was $9.56 \%$. This reflects findings from several previous studies in the literature $[5,17,18]$. The median time between the first and contralateral hip fracture was three years. For this reason, in our study, group B was divided into two subgroups with a cut-off of three years of re-fracture time. Studies from Guy et al. and others report similar rates $[6,9]$. In five out of the 37 patients, the contralateral hip fracture occurred within six months (range 12-144 days) after the first event, a period during which gait rehabilitation and functional recovery had not yet been completed. Therefore, the failure to rehabilitate and the second traumatic event might be related.

A statistically significant difference was found between the average age at first hip fracture in each group. Particularly, the average age of patients who had re-fractured within three years was significantly above the average age of those who re-fractured later. This confirms that age not only plays 
an important role in promoting a contralateral hip fracture, as already stated by several authors and confirmed by Zhu Y et al. [11] in their meta-analysis, but it also represents a risk factor that favors a shorter re-fracture time for contralateral hip fracture.

In our study, most of the patients with a proximal femoral fracture were female $(71.14 \%)$. This difference was even more marked in patients with contralateral hip fractures $(76.67 \%)$. This value could be related to a longer life expectancy in female patients. Similarly, Gaumetou et al. [9] confirmed in their study a clear predominance of first and contralateral hip fractures in females. In patients with a contralateral hip fracture within three years there was a statistically significant predominance of the male sex (average age: $86 \pm 9$ ) compared to the female sex (average age: $88 \pm 5$ ). Therefore, male patients have a shorter re-fracture time in cases of contralateral hip fracture. Additional studies with larger samples of patients could be used to perform a multivariate analysis and assess whether this result can be confirmed.

Contralateral hip fractures were often at the same anatomical site as the first fracture. Previous studies have reported between 66.6 and $80.8 \%$ correspondence [7, 9, 19], whereas in our case study a correspondence of $51.35 \%$ was found. The main morphological criterion affecting fracture site could be the length of the femoral neck: A short neck under $5 \mathrm{~cm}$ may favor the onset of a trochanteric fracture, while a neck longer than $5 \mathrm{~cm}$ may be more likely to cause an intracapsular fracture [9]. In cases of asymmetry, the contralateral hip fracture was more frequently extra-capsular, usually treated with an intramedullary nail, according to Shabat et al. This study found a pronounced decrease in bone mass in patients with extra-capsular fractures [19]. This demonstrates an elongated re-fracture time for patients with medial femoral fractures compared to lateral fractures, but this difference was not statistically significant. We hope that future studies further analyse the role of the anatomic pattern of the first hip fracture episode in the occurrence of a short-term contralateral hip fracture.

An interesting finding was a statistically significant difference in re-fracture time for patients with different PPI values. Dividing patients with contralateral hip fracture according to the re-fracture time, we found that patients with a PPI value $\geq 5$ had a significantly shorter re-fracture time compared to those with a lower PPI value. This may be related to the patient's mobility and therefore to a higher risk of falls. Orthopedic surgeons are often the first to treat these patients after a fragility fracture and, because the incidence continues to rise, they may play a more central role in the care of osteoporotic patients [20]. Early diagnosis of a fragility fracture and intervention with medication for osteoporosis is crucial for the prevention of secondary fractures. Although many anti-osteoporosis medications can reduce the risk of subsequent fractures, most patients with a fragility fracture are not treated. Furthermore, patients who use anti-osteoporosis medications often do not adhere properly to the therapy, compromising treatment effectiveness in postoperative management [21]. Patients are frightened by complications of medical therapy (atypical femoral fractures, osteonecrosis of the jaw, myocardial infarction), despite the low incidence of these complications compared with consequences of nonadherence. The use of anti-osteoporosis medications results in an estimated 5-50\% reduction in subsequent fracture risk, therefore failing to take medication after a first hip fracture can contribute to an increase in the incidence of a second fracture. Despite several studies showing the importance of preventing fractures due to fragility and second hip fractures, our data show that anti-osteoporotic drugs do not affect the time of second fracture [10, 20-22]. Similarly, other clinical and epidemiological parameters analyzed such as comorbidities, increased surgical timing during the first event and not returning to the original residence, although correlated in the literature with increased risk of contralateral hip fracture and early mortality $[10,11]$, appeared not to be related to reduced re-fracture timing. Further studies are necessary to confirm the correlation between the parameters analyzed in this study and the contralateral re-fracture timing. Moreover, it may be useful to start studies evaluating biochemical factors already related in the literature with bone healing $[23,24]$ to investigate their possible role in contralateral hip fracture occurrence and re-fracture timing.

Our study has some limitations. The design of the study is retrospective and the sample size was small for powerful statistical analysis. Therefore, a multiple logistic regression analysis was not performed because the number of observations was insufficient. Moreover, the clinical significance of mortality rate results was limited because our study had a short follow-up period. Nevertheless, the clinical series was homogeneous with many consecutive patients enrolled in our study, that were operated on by the same surgical team.

\section{Conclusion}

A multidisciplinary approach to the treatment of contralateral hip fracture should be employed. This study reveals that several risk factors play a crucial role in hip re-fracture time in patients over 65 years old. Older age, male sex and a PPI value $\geq 5$ between the first and second hip fracture are risk factors associated with a contralateral hip fracture within three years of the first episode. There were no statistically significant differences in re-fracture time regarding anti-osteoporotic therapy, anatomic site of the first hip fracture episode, increased surgical timing during the first 
event, type of comorbidities and not returning to the original residence.

\begin{abstract}
Authors' contributions FB and JV performed the literature research, drafted the manuscript, collected the data, derived the results and prepared the tables. AM, EG, MT and SA were involved in the conception and design of the study, oversaw and provided quality assurance on all study output and assisted in revising the manuscript. All the patients included in this study were operated on by the groups directed by AM All authors read and approved the final manuscript.
\end{abstract}

Funding Open access funding provided by Università degli Studi di Torino within the CRUI-CARE Agreement.

Data availability The dataset analyzed in this study is available from the corresponding author on reasonable request.

Code availability Statistical analysis was performed using the Statistical Product and Service Solutions (SPSS Inc., Chicago, Illinois).

\section{Declarations}

Conflict of interest The authors declare that they have no conflict of interest.

Ethical approval All procedures performed in studies involving human participants were in accordance with the ethical standards of the institutional and/or national research committee and with the 1964 Helsinki Declaration and its later amendments or comparable ethical standards. All patients were informed about the study and consented to participate.

Consent to participate All patients were informed about the study and consented to participate

Consent to publication All patients gave consent to publication.

Open Access This article is licensed under a Creative Commons Attribution 4.0 International License, which permits use, sharing, adaptation, distribution and reproduction in any medium or format, as long as you give appropriate credit to the original author(s) and the source, provide a link to the Creative Commons licence, and indicate if changes were made. The images or other third party material in this article are included in the article's Creative Commons licence, unless indicated otherwise in a credit line to the material. If material is not included in the article's Creative Commons licence and your intended use is not permitted by statutory regulation or exceeds the permitted use, you will need to obtain permission directly from the copyright holder. To view a copy of this licence, visit http://creativecommons.org/licenses/by/4.0/.

\section{References}

1. Bhandari M, Swiontkowski M (2017) Management of acute hip fracture. N Engl J Med 377(21):2053-2062

2. Vigni GE, Bosco F, Cioffi A, Camarda L (2021) Mortality risk assessment at the admission in patient with proximal femur fractures: electrolytes and renal function. Geriatr Orthop Surg Rehabil 2021(12):2151459321991503
3. Kesmezacar H, Ayhan E, Unlu MC, Seker A, Karaca S (2010) Predictors of mortality in elderly patients with an intertrochanteric or a femoral neck fracture. J Trauma: Injury, Infection, and Critical Care 68(1):53-158

4. Balasubramanian A, Zhang J, Chen L, Wenkert D, Daigle SG, Grauer A et al (2019) Risk of subsequent fracture after prior fracture among older women. Osteoporos Int 30(1):79-92

5. Lawrence TM, Wenn R, Boulton CT, Moran CG (2010) Agespecific incidence of first and second fractures of the hip. J Bone Joint Surg Br 92(2):258-261

6. Ryg J, Rejnmark L, Overgaard S, Brixen K, Vestergaard P (2009) Hip fracture patients at risk of second hip fracture: a nationwide population-based cohort study of 169,145 cases during $1977-$ 2001. J Bone Miner Res 24(7):1299-1307

7. Sawalha S, Parker MJ (2012) Characteristics and outcome in patients sustaining a second contralateral fracture of the hip. J Bone Joint Surg Br 94(1):102-106

8. Berry SD, Samelson EJ, Hannan MT, McLean RR, Lu M, Cupples LA et al (2007) Second hip fracture in older men and women: the Framingham Study. Arch Intern Med 167(18):1971-1976

9. Gaumetou E, Zilber S, Hernigou P (2011) Non-simultaneous bilateral hip fracture: epidemiologic study of 241 hip fractures. Orthop Traumatol Surg Res 97(1):22-27

10. Juhász K, Boncz I, Patczai B, Mintál T, Sebestyén A (2016) Risk factors for contralateral hip fractures following femoral neck fractures in elderly: analysis of the Hungarian nationwide health insurance database. Eklem Hastalik Cerrahisi 27(3):146-152

11. Zhu Y, Chen W, Sun T, Zhang Q, Cheng J, Zhang Y (2014) Metaanalysis of risk factors for the second hip fracture (SHF) in elderly patients. Arch Gerontol Geriatr 59(1):1-6

12. Kristensen MT, Foss NB, Ekdahl C, Kehlet H (2010) Prefracture functional level evaluated by the new mobility score predicts in-hospital outcome after hip fracture surgery. Acta Orthop 81(3):296-302

13. Viberg B, Kold S, Brink O, Larsen MS, Hare KB, Palm H(2020) Is arthroplasty better than internal fixation for undisplaced femoral neck fracture? A national pragmatic RCT: the SENSE trial BMJ Open 10(10):e038442

14. Parker MJ, Palmer CR (1993) A new mobility score for predicting mortality after hip fracture. J Bone Joint Surg Br 75(5):797-798

15. Voeten SC, Nijmeijer WS, Vermeer M, Schipper IB, Hegeman JH (2020) DHFA Taskforce study group Validation of the fracture mobility score against the Parker mobility score in hip fracture patients. Injury 51(2):395-399

16. Pedersen TJ, Lauritsen JM (2016) Routine functional assessment for hip fracture patients. Acta Orthop 87(4):374-379

17. Scaglione M, Fabbri L, Di Rollo F, Bianchi MG, Dell'omo D, Guido G (2013) The second hip fracture in osteoporotic patients: not only an orthopaedic matter. Clin Cases Miner Bone Metab 10(2):124-128

18. Guy P, Sobolev B, Sheehan KJ, Kuramoto L, Lefaivre KA (2017) The burden of second hip fractures: provincial surgical hospitalizations over 15 years. Can J Surg 60(2):101-107

19. Shabat S, Gepstein R, Mann G, Kish B, Fredman B, Nyska M (2003) The second hip fracture - an analysis of 84 elderly patients. J Orthop Trauma 17:613-617

20. Khan AZ, Rames RD, Miller AN (2018) Clinical management of osteoporotic fractures. Curr Osteoporos Rep 16(3):299-311

21. Balasubramanian A, Tosi LL, Lane JM, Dirschl DR, Ho PR, O'Malley CD (2014) Declining rates of osteoporosis management following fragility fractures in the US, 2000 through 2009. J Bone Joint Surg Am 96(7):e52

22. Bliuc D, Nguyen ND, Nguyen TV, Eisman JA, Center JR (2013) Compound risk of high mortality following osteoporotic fracture and refracture in elderly women and men. J Bone Miner Res 28(11):2317-2324 
23. Serbest S, Tiftikçi U, Tosun HB, Kısa Ü (2017) The Irisin Hormone Profile and Expression in Human Bone Tissue in the Bone Healing Process in Patients. Med Sci Monit 23:4278-4283

24. Serbest S, Tiftikci U, Tosun HB, Gumustas SA, Uludag A (2016) Is there a relationship between fracture healing and mean platelet volume? Ther Clin Risk Manag 12:1095-1099
Publisher's Note Springer Nature remains neutral with regard to jurisdictional claims in published maps and institutional affiliations. 